\title{
Anabases
}

ANABASES Traditions et réceptions de l'Antiquité

$20 \mid 2014$

Varia

\section{William V. HARRIS (éd.), The Ancient Mediterranean Environment between Science and History}

\section{Benoît Rossignol}

\section{OpenEdition}

\section{Journals}

Édition électronique

URL : http://journals.openedition.org/anabases/5152

DOI : 10.4000/anabases.5152

ISSN : 2256-9421

\section{Éditeur}

E.R.A.S.M.E.

\section{Édition imprimée}

Date de publication : 1 novembre 2014

Pagination : 412-413

ISSN : 1774-4296

\section{Référence électronique}

Benoît Rossignol, « William V. HARRIS (éd.), The Ancient Mediterranean Environment between Science and History ", Anabases [En ligne], 20 | 2014, mis en ligne le 01 novembre 2014, consulté le 22 septembre 2020. URL : http://journals.openedition.org/anabases/5152 ; DOI : https://doi.org/10.4000/anabases 5152

Ce document a été généré automatiquement le 22 septembre 2020

(c) Anabases 


\title{
William V. HARRIS (éd.), The Ancient Mediterranean Environment between Science and History
}

\author{
Benoît Rossignol
}

\section{RÉFÉRENCE}

William V. HARRIS (éd.), The Ancient Mediterranean Environment between Science and History, Leiden-Boston, Brill, 2013, $332 \mathrm{p}$. 112 euros / ISBN 978-90-04-25343-8

1 Voilà un ouvrage où CIL (Corpus Inscriptionum Latinarum) voisine avec ENSO (El NiñoSouthern Oscillation) dans la table des abréviations. C'est annoncer un dialogue interdisciplinaire qui va, fort heureusement, plus loin que la juxtaposition. Neuf études y confrontent des spécialistes des sciences de la nature, des archéologues, des historiens pour tracer un bilan provisoire de nos connaissances sur l'environnement de la Méditerranée antique. L'ensemble est mis en perspective par l'introduction critique et méthodologique de William Harris et par la conclusion d'Andrew Wilson qui s'attache aussi à esquisser les points que le volume n'a pu aborder en détail (séismes, pollution...).

2 P. Malanima part de la limite fondamentale qu'était l'énergie pour les sociétés préindustrielles. Le déclin antique est présenté dans une perspective assez malthusienne. L'essentiel de l'énergie antique venant de l'agriculture, la question du lien avec le climat est posée, la réussite de Rome coïncide avec une situation climatique très favorable. P. Malanima donne de grands ordres d'idée, mais la question des changements, de leurs facteurs et de leurs seuils est difficile, beaucoup reste à confirmer ou infirmer. R. Veal offre un plaidoyer pour le charbon de bois, insistant sur les informations que l'on peut tirer de cet artefact encore souvent négligé en fouille. Passant en revue les techniques d'analyses, elle montre par l'exemple de Pompéi les 
apports possibles. La multiplication d'études de ce type doit permettre à terme des approches nuancées de l'économie et de l'écologie des combustibles antiques.

Ayant cerné, au sens propre, la date de La Moselle d'Ausone, M. McCormick confronte d'autres objets (le Nil, le seigle, la chaume...) à un bilan de nos connaissances climatiques dans un esprit exploratoire sans se départir d'une interrogation pertinente et précise sur la méthode, sans enthousiasme excessif non plus sur l'état de nos connaissances : le chantier ne fait que s'ouvrir, l'heure est au travail et à l'innovation, de nombreuses pistes sont suggérées. E. Cook présente le lien possible entre les précipitations en Eurasie et ENSO (La Niña), ses cartes montrent la sensibilité de régions de l'Asie centrale mais aussi de la Volga et de la Mer Noire à ENso. Le lien avancé entre de possibles sécheresses importantes $\mathrm{du}_{\mathrm{IV}}^{\mathrm{e}} \mathrm{s}$. au vi $\mathrm{e}$. et les migrations des Huns et des Avars est plus incertain, les questions historiques (où étaient les Huns? Quelles motivations déterminaient leurs mouvements ?) sont trop négligées. Le long article de S. Manning constitue le plat de résistance de l'ouvrage. Il faut le lire en regard de l'article dirigé par M. McCormick et U. Büntgen dans le Journal of Interdisciplinary History (2012). Manning établit un bilan de nos connaissances, et surtout de leurs limites, quant au climat méditerranéen antique, en particulier pour l'époque de l'empire romain. Malgré les incertitudes et les débats possibles, le cœur de la période romaine apparaît caractérisé par une stabilité remarquable et un climat chaud et humide qui semble avoir été profitable. Cette particularité de la période qui correspond au Haut-empire revient dans d'autres communications.

4 Après son article dans les Annales (2011), W. Harris revient sur la question de la déforestation, insistant sur les questions de méthode et l'importance de la définition des concepts. Si l'impact des sociétés antiques (croissant jusqu'aux Sévères) est certain et étendu, la bonne gestion des forêts existait aussi. Les hypothèses extrêmes sont récusées pour une approche nuancée et prudente.

5 P. Kouki, avec l'exemple de la région de Pétra, montre les limites de la documentation et l'impossibilité d'une conclusion assurée quant à l'impact des changements climatiques sur les implantations de la région. Elle attire aussi à raison l'attention sur la nécessité d'éviter les catégories trop simplistes de climat favorable ou défavorable. E. Ermolli, P. Romano et M.R. Ruello abordent par deux cas italiens la difficile question de la sédimentation et de ses causes - anthropiques et/ou climatiques. Ils montrent comment la rencontre de facteurs humains et de variations du climat entraîne, lors d'une "fenêtre d'opportunité ", la survenue d'épisodes d'érosion sévère, marquant l'évolution du paysage qu'il faut aussi replacer dans la longue durée. Se consacrant aux question hydrologiques D. Keenan-Jones part de deux études témoignant de l'habileté romaine dans le domaine et de son impact, les considérant aussi au regard des représentations philosophiques, politiques ou religieuses. La continuité et la réception de cette histoire antique de l'hydrographie est esquissée jusque pour l'Italie contemporaine, dépeignant un cas remarquable de dialogue entre Antiquité et modernité.

6 Le volume est édité de manière soignée (malgré quelques coquilles dans l'article de E. Cook). Il présente de nombreuses figures et graphiques et un index. Tous les auteurs insistent sur l'ampleur de ce qui reste à connaître et l'importance des nouvelles données déjà acquises. Les articles ont des approches très différentes, de très grandes perspectives voisinent avec des études de cas plus ponctuels. Plusieurs fois des divergences apparaissent, ainsi à propos des forçages volcaniques on pourra comparer 
M. McCormick (p. 70) avec S. Manning (p. 154-156). L'ouvrage a quelques déséquilibres dans sa composition et quelques lacunes bibliographiques (l'oubli de P. Leveau surprend). Toutefois, il livre l'état des connaissances avec leurs débats et leurs incertitudes, ainsi que les pistes d'un champ qui en est encore, comme plusieurs auteurs le soulignent, à l'étape de l'expérimentation et rejoint les préoccupations des sociétés contemporaines. Un jalon important dans un domaine appelé à se développer.

\section{AUTEURS}

\section{BENOÎT ROSSIGNOL}

Université Paris 1 Panthéon-Sorbonne

Benoit.Rossignol@univ-paris1.fr 